\title{
Changes to MFRSRCLDOD1MIN Datastream
}

S McFarlane

Y Shi

May 2012

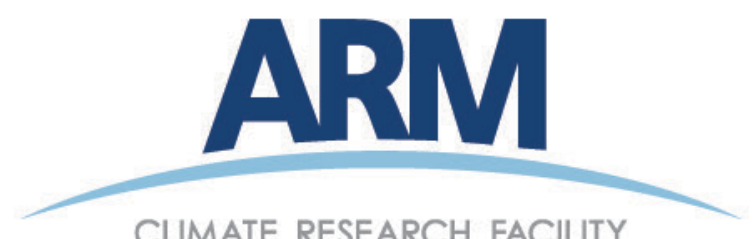




\section{DISCLAIMER}

This report was prepared as an account of work sponsored by the U.S. Government. Neither the United States nor any agency thereof, nor any of their employees, makes any warranty, express or implied, or assumes any legal liability or responsibility for the accuracy, completeness, or usefulness of any information, apparatus, product, or process disclosed, or represents that its use would not infringe privately owned rights. Reference herein to any specific commercial product, process, or service by trade name, trademark, manufacturer, or otherwise, does not necessarily constitute or imply its endorsement, recommendation, or favoring by the U.S. Government or any agency thereof. The views and opinions of authors expressed herein do not necessarily state or reflect those of the U.S. Government or any agency thereof. 


\section{Changes to MFRSRCLDOD1MIN Datastream}

S McFarlane

Y Shi

May 2012

Work supported by the U.S. Department of Energy,

Office of Science, Office of Biological and Environmental Research 


\section{Contents}

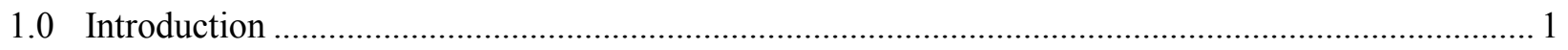

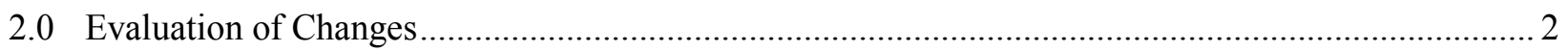

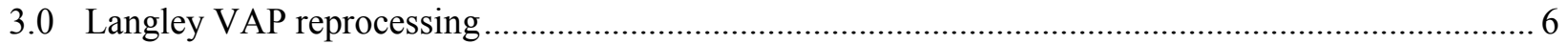




\section{Figures}

Figure 1. (left) Comparison of new and original transmittance values. (right) Difference in transmittance as a function of original transmittance value

Figure 2. Comparison of optical depth (left) and liquid water path (right) for times with transmittance values within valid range, optical depth retrieved, and lwp_source $=2$ in both original and new files

Figure 3. Original and new LWP for times when original lwp_source is from MWRLOS and new lwp_source is MWRRET.

Figure 4. Original and new effective radius for points where original data used MWRLOS and new data use MWRRET as input liquid water path.

Figure 5. Histogram of optical depth for all cases (left) and cases with cloud fraction $>0.9$ (right). Original data are plotted in black and new data in blue 


\subsection{Introduction}

Significant updates were made to the multifilter rotating shadowband radiometer (MFRSR) cloud optical depth (MFRSRCLDOD) value-added product (VAP) in 2011. The original intent of the update was to add quality control (QC) flags and to update the VAP to use the improved retrievals of liquid water path (LWP) from the Microwave Radiometer Retrievals (MWRRET) VAP rather than the statistical retrievals of LWP from the Microwave Radiometer Line of Sight (MWRLOS) datastream. Although this was originally intended to be a straightforward update of the code, it became more complicated due to the following factors:

- a new developer and translator team were working with the code

- numerous small changes had to be made to the code to consistently implement the QC flags

- ARM standards have changed over the years since the code was originally developed.

This document does not give details of every change that was made to the VAP, but documents the primary changes:

1. When available, VAP now uses liquid water path from MWRRET, rather than MWRLOS, to retrieve effective radius. If available, the "be_lwp" variable from MWRRET is used. If this variable is $\mathrm{bad} / \mathrm{missing}$, then "phys_lwp" is used if good or indeterminate. If it is not available, then "stat2_lwp" is used if good or indeterminate. If all are missing/bad, then the default effective radius $=8.0 \mathrm{um}$ is used. The value of lwp_source is set to 3 if the LWP measurement comes from the MWRRET datastream.

2. The MFRSRCLDOD1MIN datastream was changed to contain data from 0000 to 2400 UTC to be consistent with other ARM datastreams and to simplify operation when we move to new sites. (Previously, the datastream was run on a solar day, 0600 to 0600 UTC, at the Southern Great Plains).

3. The Langley VAP, which is an input to MFRSRCLDOD, has been updated to correct for the MFRSR calibration before calculating the Io and to output the Io in counts instead of $\mathrm{W} / \mathrm{m} 2 / \mathrm{nm}$. At this point only a portion of the historical data has been reprocessed. The VAP was updated to check the units of Io and treat the data accordingly. However, since the MFRSRCLDOD reads in several months of Io data at one time to find the best Io to use for the given day, a 4-month period centered on the time of transition between Langley versions at each site is not being processed. When the Langley data at a given site are reprocessed, the MFRSRCLDOD will also be reprocessed. A list of dates for the various Langley versions at each site is attached at the end of this document.

4. Quality control flags were added to the VAP output. Details for reading bit-packed QC flags can be found at: https://engineering.arm.gov/ shippert/ARM_bits.html. Below are some details of how the QC flags may affect the output variables.

a. valid_min and valid_max values were added to each input and output variable; values outside of these ranges are flagged as bad and set to -9999

b. As the retrieval is only valid for overcast skies, the cloud fraction (cf) from the shortwave flux analysis is used to assess the validity of the retrieval. Periods with cf between 0.7 and 0.9 are flagged as indeterminate. Periods with cloud fraction $<0.7$ are flagged bad, and retrieved data are set to -9999 . 
c. When an input lwp value is not available (because it is bad/missing or the site does not have an MWR), the default effective radius of $8.0 \mathrm{um}$ is used, and the effective radius is flagged as indeterminate.

d. The VAP estimates errors in the retrieved effective radius and optical depth by perturbing the input values slightly and re-running the retrieval. For cases where the default effective radius is used, certain of these error terms cannot be calculated. In these cases the error terms are flagged bad, and the values are set to -9999 . For sites that do not have microwave radiometers, these error terms will always be set to -9999 .

e. Along with instantaneous values, the VAP reports values of optical depth, effective radius, and error terms averaged over a 5-min period. If any of the instantaneous values within that 5-min period are bad, then the average values for the period are flagged as bad and set to -9999. If any of the instantaneous values used in the average are indeterminate, then the average value is also flagged as indeterminate.

5. Changes were made to the data object design (DOD). Numerous small changes were made to the netCDF header, including fixes in variable names (i.e. 'optical_depth_instaneous' was corrected to 'optical_depth_instantaneous'), and other changes required to meet new ARM DOD standards were made.

6. Quicklook plots were updated so that names follow new ARM standards.

7. Since the original MFRSRCLDOD1MIN data were processed, the MFRSR datastream was reprocessed. Therefore, some changes between previous/new versions of MFRSRCLDOD1MIN are due to changes in the MFRSR datastream.

8. Based on the end-to-end MFRSR reprocessing, we intend to apply the MFRSRCLDOD1MIN VAP to all historical MFRSR data available (previously only data 12/2004 and later were processed).

\subsection{Evaluation of Changes}

We examined one year of data (20050101-20051231) at the SGP E13 site to evaluate the effect of changes in the MFRSRCLDOD VAP on the data.

Changes in Transmittance: To evaluate the effect of changes in the input MFRSR data on the retrieval, we examined the 195,786 times where both the old and new files had transmittance between 0 and 1 .

- Mean original transmittance: 0.202

- Mean new transmittance: 0.211

- Mean difference (new - orig): -0.008

- RMS difference: 0.005

In general the new MFRSR data have slightly higher transmittance values than the original data. Although these average transmittance differences seem quite small, the plot below (Figure 1) shows that large differences in optical depth can result from small changes in transmittance at small transmittance values. 

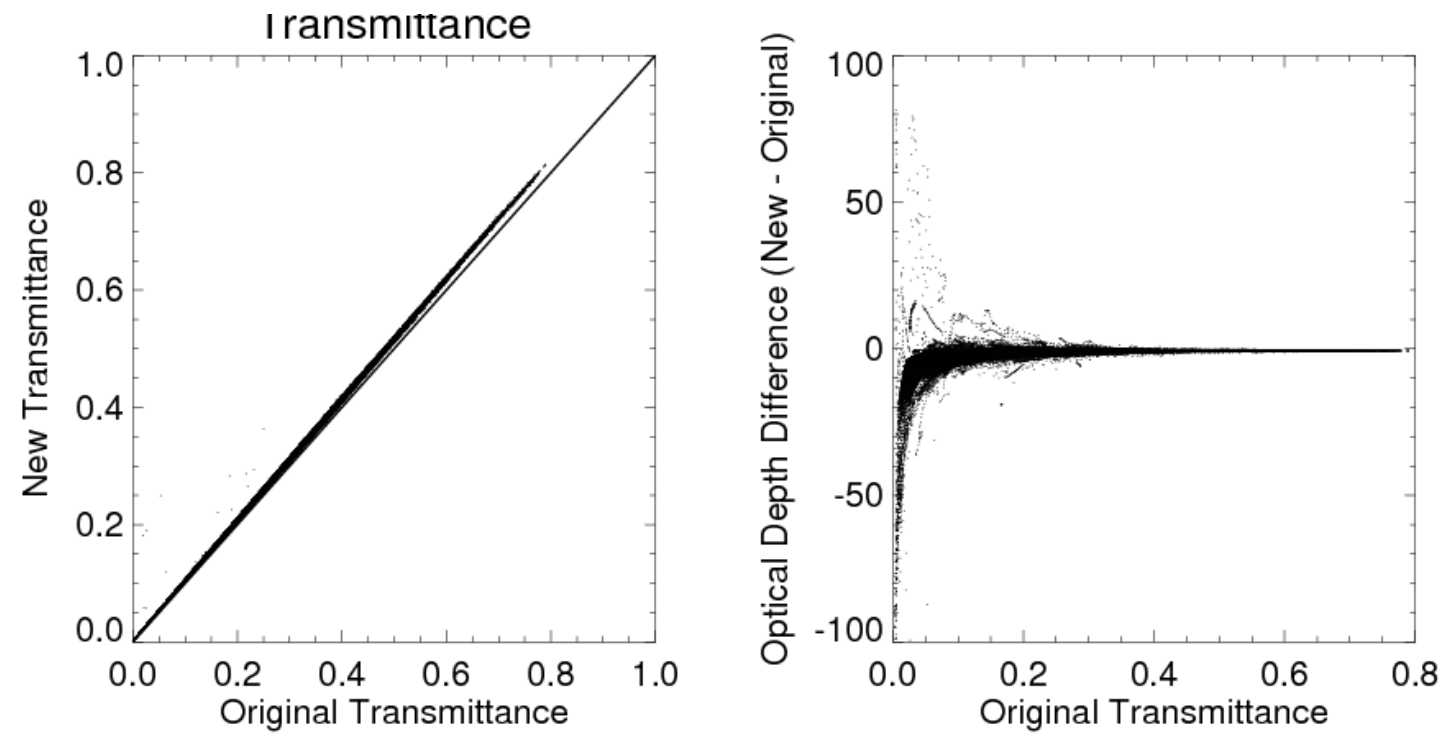

Figure 1. (left) Comparison of new and original transmittance values. (right) Difference in transmittance as a function of original transmittance value.

Figure 2 shows the retrieved instantaneous optical depth and liquid water path derived from the MFRSR for all times where transmittance is within valid range in both old and new files, optical depths are retrieved in both files, and lwp_source $=2$ (LWP derived from MFRSR). Associated with the higher transmittance values, the new data tend to have smaller optical depths. The mean instantaneous optical depth values for these 114,184 points are:

- Mean original tau: 19.62

- Mean new tau: 17.78

- Mean difference (new-orig): -1.84

- RMS difference: 5.87

- Mean original LWP: $0.106 \mathrm{~mm}$

- Mean new LWP: $0.096 \mathrm{~mm}$

- Mean difference (new-orig): $-0.010 \mathrm{~mm}$

- RMS difference: $0.031 \mathrm{~mm}$ 

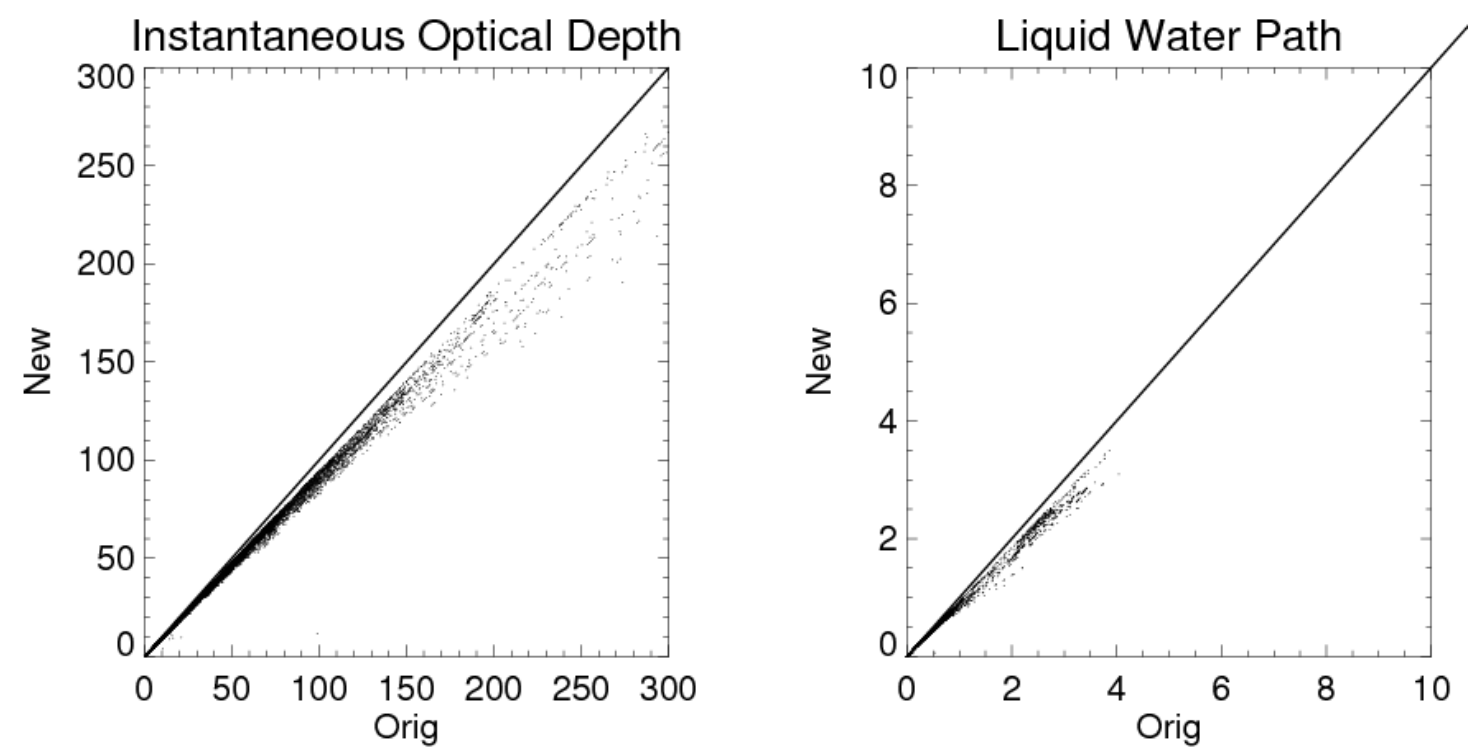

Figure 2. Comparison of optical depth (left) and liquid water path (right) for times with transmittance values within valid range, optical depth retrieved, and lwp_source $=2$ in both original and new files.

Change in LWP: In the updated version of the VAP, LWP is input from the MWRRET datastream (when available), rather than the MWRLOS datastream. Figure 3 shows the original and new liquid water path for times when the original LWP is from the MWRLOS datastream and the new LWP is from MWRRET. In general, the new LWP is slightly smaller, with a mean value of $0.199 \mathrm{~mm}$ compared to the original value of $0.223 \mathrm{~mm}$. The mean difference and RMS difference are -0.022 and $0.050 \mathrm{~mm}$, respectively.

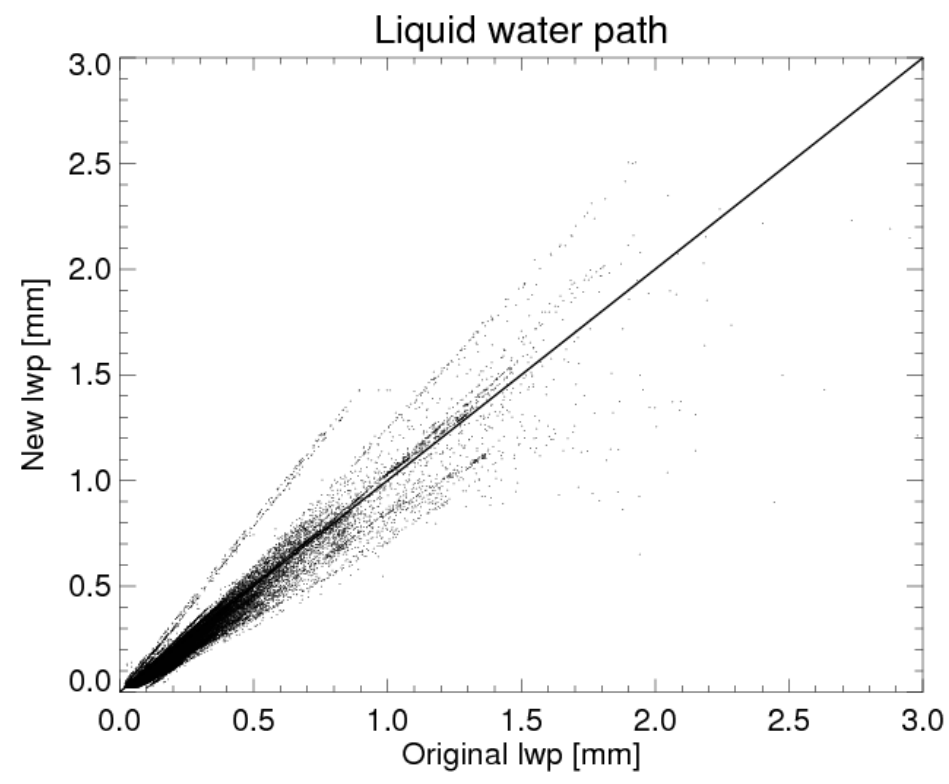

Figure 3. Original and new LWP for times when original lwp_source is from MWRLOS and new lwp_source is MWRRET. 
Change in LWP on effective radius: We cannot completely control for the effect of the change in LWP from using MWRRET instead of MWRLOS on the effective radius because of the concurrent updates in the MFRSR transmittance values. However, Figure 4 shows the original and new effective radius for points with valid retrievals in both files where the original LWP source was MWRLOS and the new LWP source is MWRRET. The original mean effective radius was $9.15 \mathrm{um}$ and the new mean is $8.80 \mathrm{um}$, for mean and RMS differences of -0.35 um and 1.74 um, respectively.

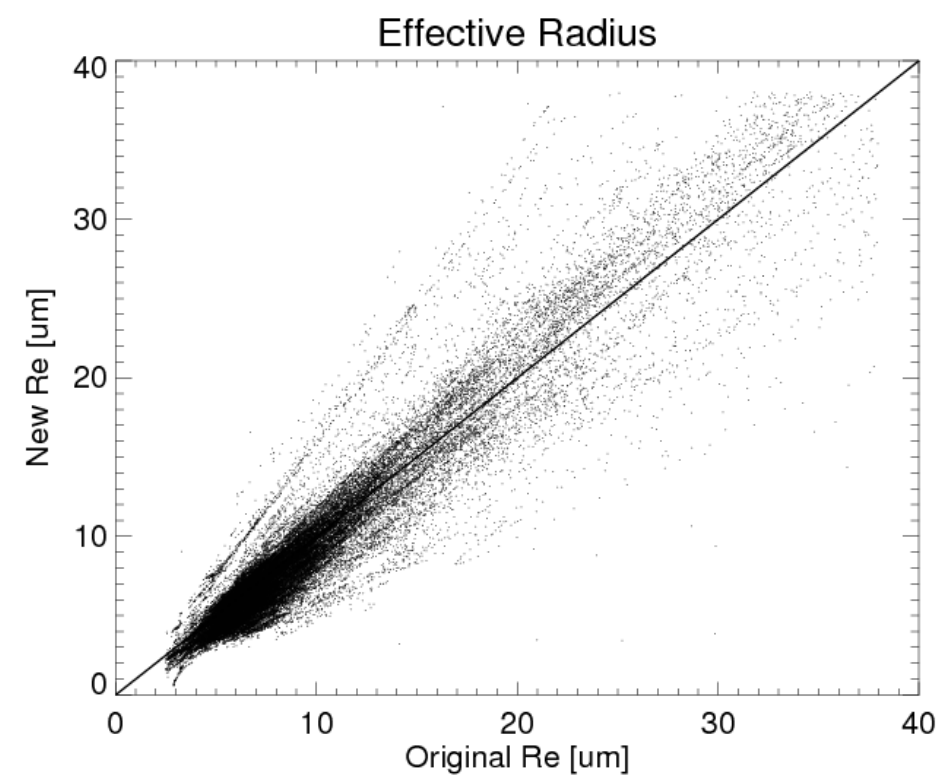

Figure 4. Original and new effective radius for points where original data used MWRLOS and new data use MWRRET as input liquid water path.

Quality control flags: The addition of quality control flags, particularly the flag on cloud fraction, removed a significant amount of data from the files. Approximately $60 \%$ of the original data that had optical depth $>0$ now have optical depth set to -9999 because of quality control flags. $80 \%$ of these times are flagged because cloud fraction is less than 0.7 and $20 \%$ of the times are flagged because transmittance is outside the expected maximum value in the look-up table for the given solar zenith angle, which represents likely broken cloud conditions.

Figure 5 shows that these cases were mostly low optical depth cases. The figure on the left shows the optical depth histogram for the original data (black) and the new data (blue) for all cases. The figure on the right shows the optical depth histogram for data where cloud fraction $>0.9$. 

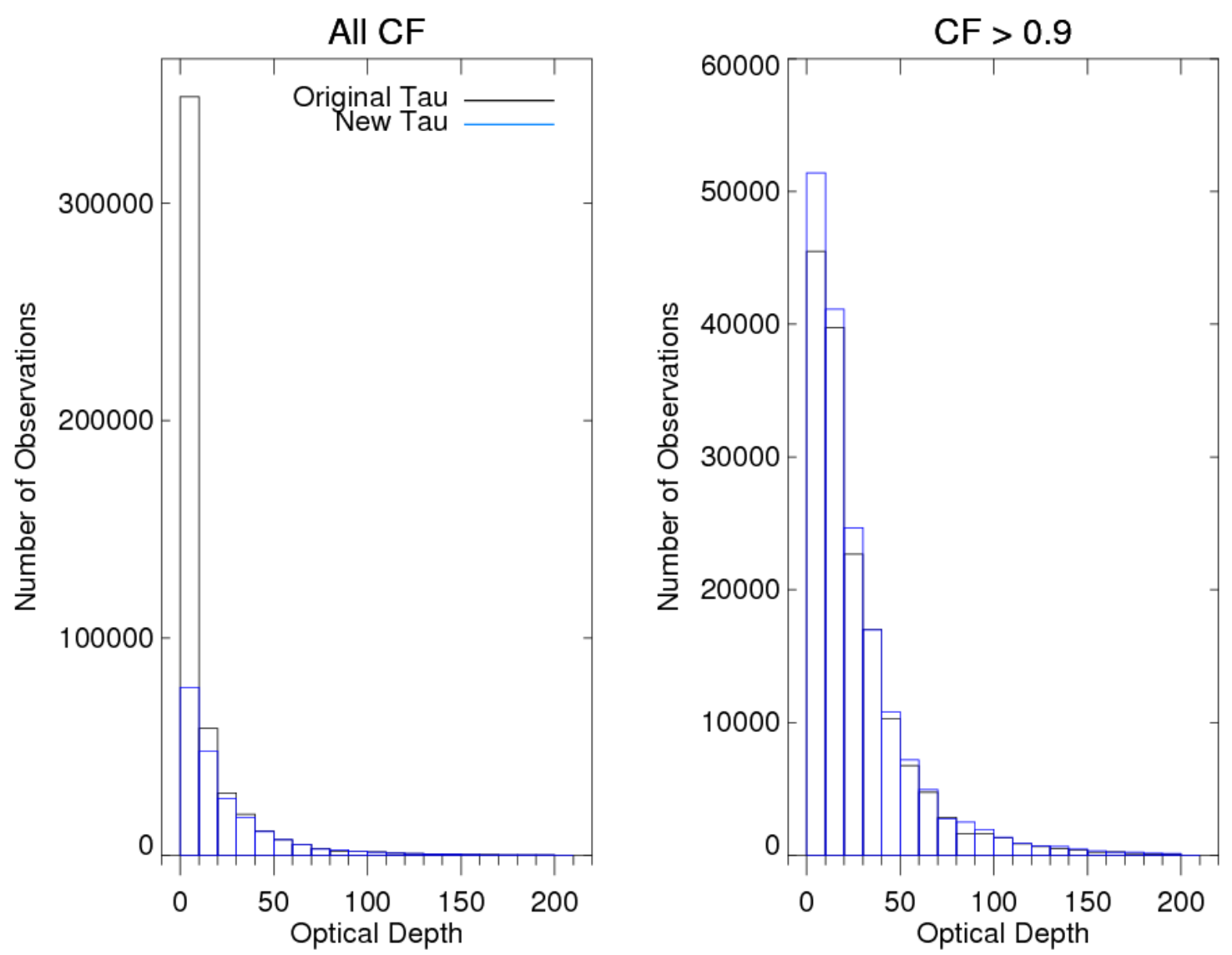

Figure 5. Histogram of optical depth for all cases (left) and cases with cloud fraction $>0.9$ (right). Original data are plotted in black and new data in blue.

\subsection{Langley VAP reprocessing}

The date ranges processed as "counts" are:

- SGP.E13 - 20090413 to present

- SGP.C1 - 20090105 to present

- SGP.E19 - all data (19980708 to present)

- SGP.E7 - all data (19971101 to present)

- TWP.C2 - 20091001 to present

For the following facilities, data were processed from 20090701 to present: SGP.E4, SGP.E6, SGP.E9, SGP.E10, SGP.E11, SGP.E12, SGP.E15, SGP.E16, SGP.E20, SGP.E27, SGP.C1 NIMFR, TWP.C1, TWP.C3, NSA.C1 (MFRSR and NIMFR), and NSA.C2 (MFRSR and NIMFR).

The following SGP facilities were decommissioned in late 2009, and none of their data were processed with the new format: E1, E2, E3, E5, E8, E18, E22, and E24. SGP.E25 was likewise decommissioned in 2002, and none of the Langley data were processed with the new format. 


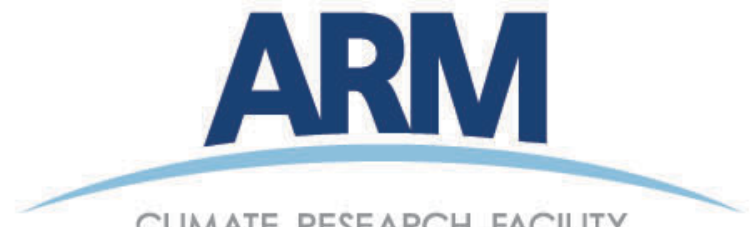

CLIMATE RESEARCH FACILITY

www.arm.gov

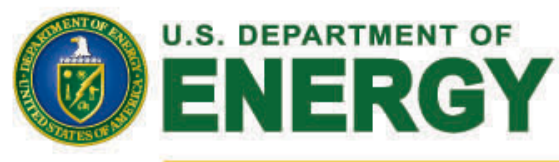

Office of Science 